\title{
Optical gain characterization of nano-ridge amplifiers epitaxially grown on a standard $\mathrm{Si}$ wafer
}

\author{
Yuting Shi ${ }^{1}$, Bernardette Kunert ${ }^{2}$, Marina Baryshnikova ${ }^{2}$, Marianna Pantouvaki ${ }^{2}$, Joris \\ Van Campenhout ${ }^{2}$, Dries Van Thourhout ${ }^{1,2}$ \\ ${ }^{1}$ INTEC Department, Ghent University,Technologiepark-Zwijnaarde 15, 9052 Ghent, Belgium \\ ${ }^{2}$ IMEC, Kapeldreef 75, 3001 Heverlee, Belgium \\ yuting.shi@ugent.be
}

\begin{abstract}
With this paper, the authors report optical gain characterization of InGaAs/GaAs nano-ridge amplifierss epitaxially grown on a standard 300-mnm Si wafer, by varying strip length (VSL) method. The measured material gain is $\sim 4000 \mathrm{~cm}^{-1}$, which is comparable with conventional GaAs material.
\end{abstract}

OCIS codes: $140.3380,160.6000,160.4236$

\section{Introduction}

The direct epitaxy of III-V semiconductors on silicon is considered as the ultimate strategy for large-scale integration of active device with passive Si photonics circuit and as such has attracted great interest in the last years. We recently demonstrated lasing under pulsed optical pumping from InGaAs/GaAs nano-ridge devices, grown on standard silicon wafers using a selective epitaxial growth process [1]. Reliable laser operation requires good crystalline quality as material defects might reduce the gain, resulting in increased threshold, excessive heat generation and limited device lifetime. Although techniques like SEM, STEM or AFM provide first feedback on the material quality, they do not provide information on the optical performance. In most cases the latter is determined from PL measurements or realizing laser devices and determining their threshold pump level. This provides only limited information on the gain properties of the grown layers however. Therefore, here we use a variable stripe length (VSL) method to extract the optical gain of GaAs nano-ridges over a wide range of pumping conditions.

\section{InGaAs/GaAs nano-ridge structure}

The epitaxy process used to grow the InGaAs/GaAs nano-ridges relies on the aspect ratio trapping (ART) effect to inhibit defects from extending into the active layers. The detailed process is described elsewhere [2]. It starts from narrow oxide trenches defined on a standard (001) Si wafer in which a GaAs buffer layer is grown using a metalorganic chemical vapor deposition (MOCVD) process. The shape and size of the nano-ridge are controlled through optimizing the growth conditions, such that it can support low loss optical mode with high optical confinement in the embedded InGaAs QWs. An InGaP passivation cap is grown around the GaAs nanoridge as a passivation layer. The
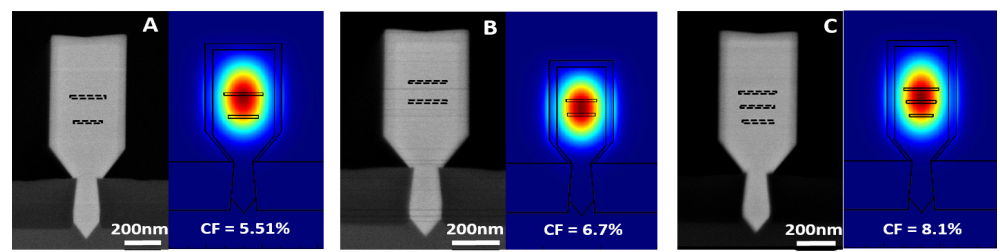

Fig. 1. Cross-section SEM image of the three nano-ridges and their corresponding calculated basic TE mode by FDE) simulation.

basic TM-like mode of the nano-ridge is rather extended in vertical direction and exhibits a high leakage loss towards the Si substrate. Also the higher order TEM modes existing in the nano-ridge waveguide suffer from high leakage loss and low QWs confinement. Therefore, we believe the basic TE mode $\left(\alpha_{\text {leak }}<5 \mathrm{~dB} / \mathrm{cm}\right)$ to be the dominant optical 
mode in the nano-ridge waveguide. Three samples, with different nano-ridge cross-section where evaluated. Samples A and B contain 2 QWs, while sample C contains 3 QWs. Sample B has a smaller overall size (height $638 \mathrm{~nm}$ ) than sample A and B (height $750 \mathrm{~nm}$ ). These variations lead to important differences in the optical confinement factor (CF) for the basic TE-mode. Fig.1 shows SEM pictures of the respective nano-ridges and the corresponding basic TE mode calculated using a finite difference eigenmode (FDE) simulation, indicating also the CF. The CF obviously increases for an increased number of QWs but also if the QWs are placed closer to the center of the nano-ridge (sample B).

\section{Gain characterization by VSL}

A Nd:YAG nanosecond pulsed laser ( $7 \mathrm{~ns}$ pulse width, $938 \mathrm{~Hz}$ repetition rate, $532 \mathrm{~nm}$ wavelength) is used for pumping the nano-ridges through the top surface. A variable rectangular slit, inserted in the setup allows to vary the length of the pumped region from 10 to 200 mum. The pump intensity is controlled by a combination of a polarizer and a rotating half-wave plate (HWP). The amplified spontaneous emission (ASE) is collected through a lensed fiber coupled to a cleaved facet of the nano-ridge and measured with an optical power meter. The raw experimental data is shown in Fig. 2 (a), as function of the pump length and for different surface illuminated pump intensities. For a given pump intensity, the measured signal is dominated by spontaneous emission (SE) for short pump lengths and then overtaken by ASE with increasing pump length. The signal saturates when further increasing then pump length. The whole pump length depend process is described by the equation: $g_{m} \cdot L=\alpha\left(I-I_{0}\right)+\ln \left[\beta\left(I-I_{0}\right)+1\right]$, with $g_{m}$ the modal gain, $L$ the pump length, $I_{0}$ the background noise, $\alpha$ describing the gain saturation process and $\beta$ a parameter related to the spontaneous emission. By fitting the experimental data to this equation, the modal gain $g_{m}$ can be extracted. $g_{m}$ can be associated also to the slope of linear region (ASE regime) in the plot from Fig.2 (a). By repeating this procedure at for all pump intensities, the pump intensity dependent modal gain can be obtained, as plotted in Fig.2 (b) for all three samples. The results show a strong correlation between optical confinement factor in the QW region and the maximum modal gain obtained. Taking into account the confinement factor and a moderate waveguide loss, the material gain can be estimated to be above $4000 \mathrm{~cm}^{-1}$.
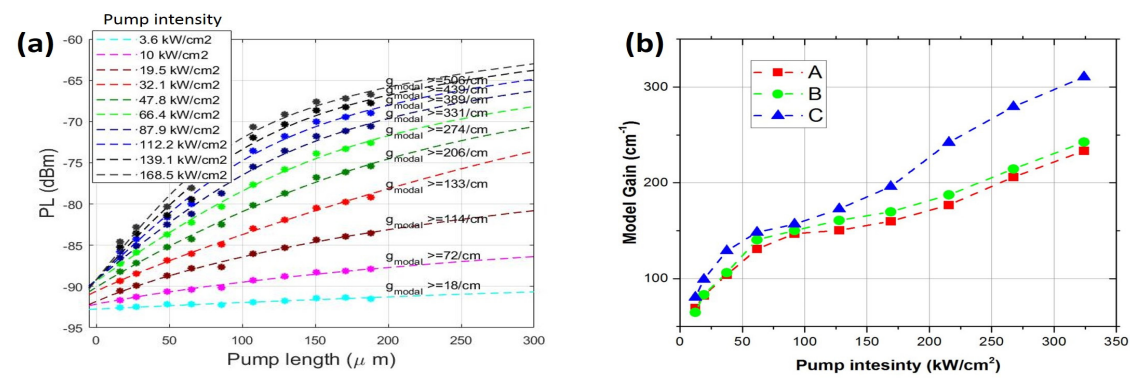

Fig. 2. (a) Experimental data of measured ASE as function of the pump length and for different pump intensities. (b) Fitted modal gain $g_{m}$ as a function of pump intensity for sample A, B and C.

\section{Conclusion}

In this paper, we report optical gain characterization of InGaAs/GaAs nano-ridge amplifierss epitaxially grown on a standard 300- mm Si wafer, using a variable stripe length method. The measured modal gain is as high as $310 \mathrm{~cm}^{-1}$. Taking into account a QW confinement factor around $8 \%$ for the basic TE-mode, this means a material gain in the order of $4000 \mathrm{~cm}^{-1}$ is obtained, comparable with results for traditional III-V materials. The proposed characterization technique provides valuable feedback and information on the quality of the nano-ridge epitaxy process and the laser design, allowing for a shorter learning cycle and improved optimization process.

\section{References}

1. Y. SHI, Z. WANG, J. VAN CAMPENHOUT, M. PANTOUVAKI, W. GUO., B. KUNERT, A. D. V. T. (2017). Optical pumped InGaAs / GaAs nano-ridge laser epitaxially grown on a standard 300-mm Si wafer. Optica, 4(12).

2. B, Kunert, W. Guo, Y. Mols, B. Tian, Z.Wang, Y. Shi, . III / V nano ridge structures for optical applications on patterned 300mm Silicon, (Md), 110. 